\title{
IMAGEM INFRAVERMELHA NO DIAGNÓSTICO DAS DOENÇAS DOS PÉS
}

\author{
Cláudia Maria Duarte de Sã Guimarães', Marcos Leal Brioschi ${ }^{2}$, Eduardo Borba Neves ${ }^{3}$, Luciane Fachin \\ Balbinot $^{4}$, Manoel Jacobsen Teixeira ${ }^{5}$
}

\author{
1 Pós-Graduação em Termologia Clínica e Termografia- HC-FMUSP \\ 2 Pós-Graduação em Termologia Clínica e Termografia- HC-FMUSP \\ ${ }^{3}$ Instituto de Pesquisa da Capacitação Física do Exército- IPCFEx \\ ${ }^{4}$ Pós-Graduação em Termologia Clínica e Termografia- HC-FMUSP \\ ${ }^{5}$ Departamento de Neurologia, Faculdade de Medicina da Universidade de São Paulo
}

Resumo: Este trabalho tem como objetivo apresentar a técnica de registro de imagens infravermelhas dos pés como método de avaliação neurovascular para identificar comorbidades usando um método não invasivo. Método: Durante o período de março de 2015 e fevereiro de 2017, foram avaliados 22 pacientes portadores de onicomicose crônica. 0 registro das imagens infravermelhas foi efetuado com câmera térmica Flir ${ }^{\circledR}$ modelo T420bx, FOL 18mm, resolução IR320 x 240, sob condições de controle de circulação de $\operatorname{ar}(<0,2 \mathrm{~ms})$, temperatura ambiente de $23^{\circ} \mathrm{C}$. Resultados: Foram identificadas 6 áreas de interesse de acordo com o território neurovascular de uma determinada região dos membros chamado de angiossoma, que foram estudadas com a comparação das áreas impares nas plantas do pé esquerdo e pares nas plantas no pé direito. Embora a amostragem seja pequena, observou-se estatisticamente uma tendência à onicomicose subungueal distal lateral (OSDL) nos diabéticos em relação à onicomicose distrófica total (ODT). Conclusões: 0 exame por imagem infravermelha pode avaliar precocemente alterações vasculares, ortopédicas e neurológicas e servir de ferramenta para rastrear comorbidades sistêmicas ou locoregionais dos membros inferiores.

Palavras-chave: termografia; onicomicose; pé; sistema vasomotor.

\begin{abstract}
This study aims to present the technique of recording infrared images of the feet as a method of neurovascular evaluation to identify comorbidities using a non-invasive method. Method: During the period of March 2015 and February 2017, 22 patients with chronic onychomycosis were evaluated. Infrared images recording was perfor-med with Flir ${ }^{\circledR}$ model T420bx, FOL 18mm, IR320 x 240 resolution, under air circulation control conditions $(<0.2 \mathrm{~ms})$, ambient temperature of $23^{\circ} \mathrm{C}$. Results: Six areas of interest were identified according to the neurovas-cular territory of a certain region of the limbs called angiosoma, which were studied by comparing the odd areas in the left foot and the right foot. Although sampling was small, a trend towards lateral distal subungual onycho-mycosis (OSDL) was observed in diabetics in relation to total dystrophic onychomycosis (ODT). Conclusions: Infrared imaging can assess early vascular, orthopedic and neurological changes and serve as a tool to track sys-temic or locoregional comorbidities of the lower limbs.
\end{abstract}

Keywords: thermography; onychomycosis; foot; vasomotor system. 


\section{† РAJMT INTRUDUÇÃO}

A técnica de registro da emissão de radiação infravermelha de um corpo captada por uma câmera com sensores especiais, que através de um software processam esta radiação em forma de temperatura formando os chamados termogramas. A técnica não invasiva destina-se ao registro das áreas quente e frias produzindo imagens digitais que são passíveis de análise em intervalos de temperatura que são expressos em faixas de cores. As temperaturas das áreas analisadas variam de acordo com o aporte sanguíneo local e a anormalidade clínica ${ }^{1}$. A primeira padronização do protocolo das imagens foi publicada pela The International Standards Organization em 2008². Hoje em dia as imagens digitais são processadas e analisadas em diferentes escalas de cores e, na medicina, a escala mais utilizada é a "Rainbow High Contrast ou HC" que, resumidamente, identifica o vermelho como quente e o azul como frio com uma precisão de $0,2^{\circ} \mathrm{C}$ com uma distribuição em escala linear ou logarítmica. Os termogramas também carregam as informações sobre a hora e a data na qual foram realizados, para fidedignidade das imagens, essencial para seu uso nas perícias médicas. Além disto, nos últimos 20 anos cresceu o interesse sobre a padronização dos protocolos clínicos, preparação do paciente, posições do corpo para a gravação das imagens e a avaliação da imagem térmica ${ }^{2}$. A padronização das áreas de estudo da pele vem sendo realizada paulatinamente através da identificação das ROI (region of interest, área de interesse em inglês), de acordo com a anatomia corporal e aplicação de testes, como o teste de estimulo ao frio (cold stress test, em inglês) 3,4. Um dos segmentos anatômicos de especial interesse a serem estudados são os membros inferiores. Esta parte da anatomia humana está intrinsicamente relacionada com a deambulação e o controle da reserva sanguínea periférica. Desta forma, as estruturas ósseas, musculares e neurovasculares atuam em conjunto auxiliando o equilíbrio hidrodinâmico e biomecânico no organismo. O território neurovascular de uma determinada região dos membros dá-se o nome de angiossoma ${ }^{5}$. A captura dos termogramas permite estudar de forma não invasiva processos inflamatórios vasculares ou shunt-arteriovenosos ${ }^{6}$, sobrecargas musculares, assim como a perfusão sanguínea nas regiões de interesse estudadas. O segmento anatômico dos pés reflete diversas alterações relacionadas com a marcha e doenças sistêmicas. Neste segmento, destaca-se a região plantar.

A região plantar é uma região com anatomia peculiar e pode ser dividida em angiossomas que podem ser posteriormente analisados com a termografia, inclusive de forma dinâmica com aplicação de teste de estímulo ao frio ${ }^{7}$, realizado, por exemplo nos diabéticos nos quais pode-se avaliar as alterações circulatórias e/ou neurológicas com os termogramas dos pés $8,9,10,11$. A anamnese dos pacientes com problemas nos pés deve ser dirigida para investigar causas de dores, edemas e redução da mobilidade do tornozelo. Entre as causas de dores destacam-se varizes, tromboflebite, osteoartrite, artrite reumatoide, patologias malignas, osteomielite, problemas no joelho, tendinites do tendão de Aquiles, fasceíte plantar, claudicação de origem arterial (intermitente), claudicação venosa, mialgia, neuropatia periférica e linfedema ${ }^{12}$. A dor de origem arterial desaparece com o repouso e piora com a deambulação, enquanto a dor que se origina na hipertensão venosa piora com o repouso e pode vir acompanhada de câimbras, principalmente noturnas. ${ }^{13,14}$

Entre as causas de edema e consequente sofrimento vascular da pele e subcutâneo dos pés devido ao depósito de fibrina, proteínas e polissacarídeos, destacam-se: insuficiência venosa crônica, falência cardíaca, renal, trombose ou obstrução de veias profundas, hipoalbuminemia, síndromes de retenção de fluidos, linfedema, lipodistrofia, hemihipertrofia e agenesia venosa valvular, além de problemas ortopédicos ${ }^{12,15}$. Wagner em 1981 gradua ${ }^{16}$ as lesões dos pés em seis graus, de acordo com a presença ou não de lesão cutânea, infecção ou gangrena. $O$ autor ressalta que os distúrbios circulatórios são responsáveis pela maioria das amputações e neste grupo, 50\% a 70\% são diabéticos.

Algumas neuropatias podem promover dor no tornozelo como a síndrome do túnel do tarso anterior por compressão do nervo fibular em decorrência de edema, traumatismos, fraturas ou uso de sapatos apertados, e a síndrome do túnel do tarso posterior associada a fraturas localizadas, anormalidades vasculares, diabetes mellitus, hipotireoidismo, osteoporose migratória regional, hanseníase, entre outras patologias como relata ${ }^{17}$. As dores nos pés podem ser causadas por neuropatias, tais como: neuroma interdigital (Neuroma de Morton), polineuropatias periféricas (caraterizada pela sensação de dormência, queimação ou formigamento nas extremidades).

A fisiologia dos membros inferiores está relacionada com o bom funcionamento segmentar muscular e o alinhamento postural e influenciam a circulação local. Assim como o restabelecimento da circulação arterial e venosa favorece a deambulação adequada e a redução de edemas nos pés e tornozelos. 0 estudo destas estruturas de forma dinâmica é importante para entendermos as diversas patologias e aprimorarmos as condutas tera- 
pêuticas. Taylor e Palmer em um extenso estudo anatômico realizado em 1987 cunharam o termo angiossomo. Este conceito indica o território tridimensional vascular (arterial e venoso) de camadas de tecidos entre a pele e o osso. A partir deste conceito básico estes autores estudaram de forma segmentar várias partes do corpo, perfundindo contraste radiopaco nas artérias que nutrem a pele, músculos e ossos, seguido da dissecção do material, estudo radiológico e mapeamento detalhado. Este trabalho realizado nas pernas possibilitou a criação de um modelo tridimensional no qual a irrigação dos tecidos através das artérias sural, poplítea, tibial anterior e peroneal resultou em áreas funcionais ou angiossomos nas quais leva-se em consideração as veias e a inervação local (Figuras 1 e 2). Algumas regiões são nutridas e/ou comandadas por um ou dois angiossomas nos membros superiores, contudo nos membros inferiores estas regiões são supridas por apenas um angiossoma, sobretudo no segmento anterior. ${ }^{5}$

A importância do estudo dos angiossomas foi descrita no trabalho de revascularização dos membros (by-pass ou terapia endovascular) com o intuito de evitar a perda, melhorando a qualidade de vida e prolongando a sobrevida dos pacientes com isquemia. Sabe-se que o fluxo sanguíneo presente na região corrigida dos pés proporciona uma boa cicatrização tanto nos casos de preservação do membro quanto nos casos de amputação. ${ }^{18}$
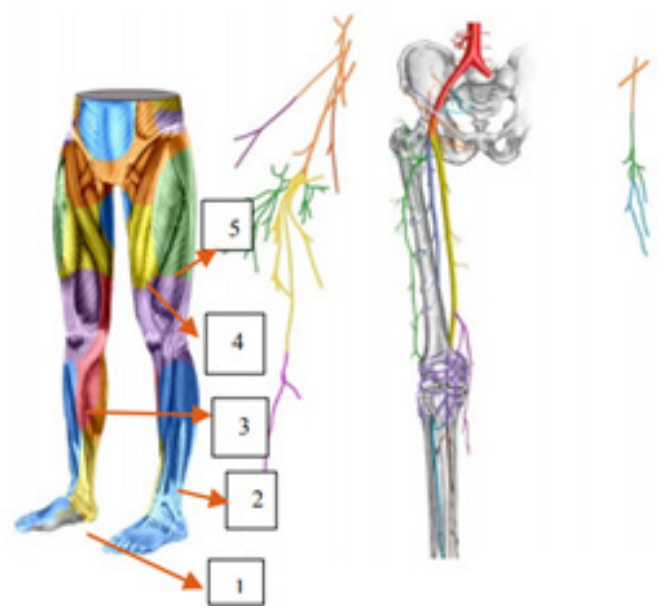

FIGURA 1 Diagrama esquemático dos angiossomos dos membros in-
feriores (vista anterior - Suami)
- Ilustração de Iriam Starling
1. artéria tibial posterior
2. artéria tibial anterior
3. artéria genicular descendente
4. artéria femoral superficial
5. artéria femoral circunflexa lateral
Outro aspecto é a insuficiência venosa crônica, caracterizada por mal funcionamento do sistema valvular com ou sem obstrução do fluxo venoso e que acomete os adultos a partir da $3^{a}$ década da vida. Este quadro muitas vezes está associado a disfunção da bomba dos músculos da panturriIha que comprime as veias profundas durante a contração, o que leva a hipertensão venosa. Esta condição facilita o acúmulo de liquido intersticial e fibrinogênio no tecido subcutâneo gerando edema, lipodermatoesclerose e ulceração. ${ }^{15} \mathrm{O}$ fortalecimento da musculatura da panturrilha e dos pés favorece a melhora do quadro venoso, o que corrobora a importância dos angiossomas (Figura 3) no estudo dos membros inferiores. A falência destes músculos pode ser decorrente de flacidez muscular, patologias neuromusculares ou tempo prolongado em pé. ${ }^{14}$

O controle vascular dos membros inferiores é realizado pelo sistema neurovegetativo simpático que regula os capilares e os shunts arteriovenosos. Este sistema promove as alterações necessárias para manter a irrigação das estruturas anatômicas, que podem ser avaliadas de acordo com a reper-

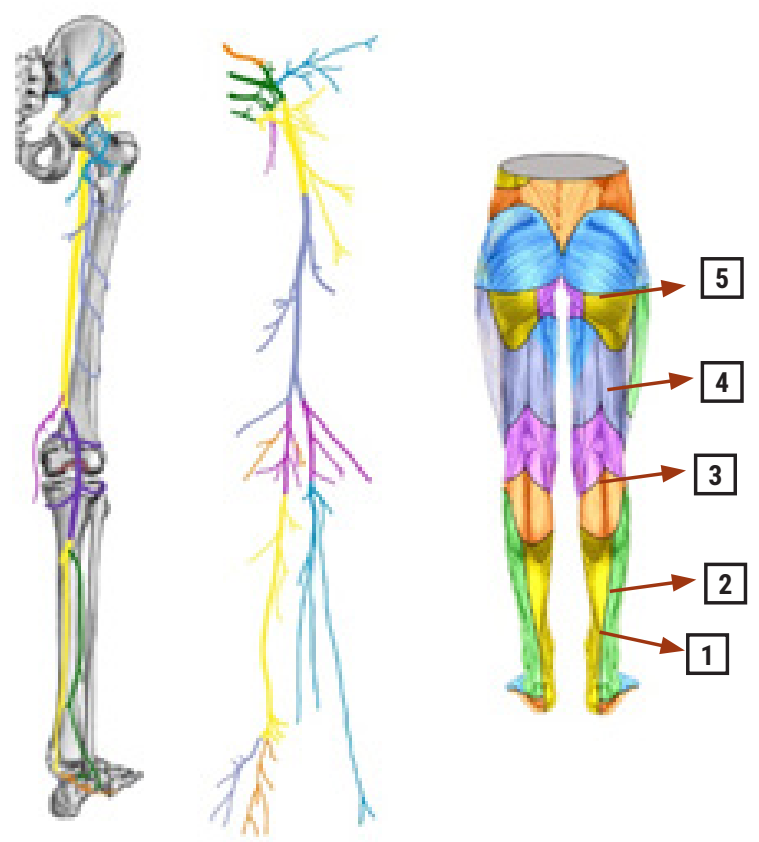

FIGURA 2 angiossomos dos membros inferiores (vista posterior modificado de Suami ${ }^{29}$

- Ilustração de Iriam Starling

1. artéria plantar lateral

2. artéria peroneal

3. artéria tibial posterior

4. artéria poplitea

5. artéria femoral profunda 


\section{PAN AMERICAN JOURNAL OF MEDICAL THERMOLOGY}

cussão da vasodilatação ou vasoconstricção na pele. O sistema simpático pode estar comprometido nos casos de neuropatia simétrica distal que pode iniciar com o acometimento das fibras finas vasomotoras, ocorrendo em 50\% dos diabéticos, carência de vitamina B12, abuso de álcool, hipotireoidismo, entre outras doenças.

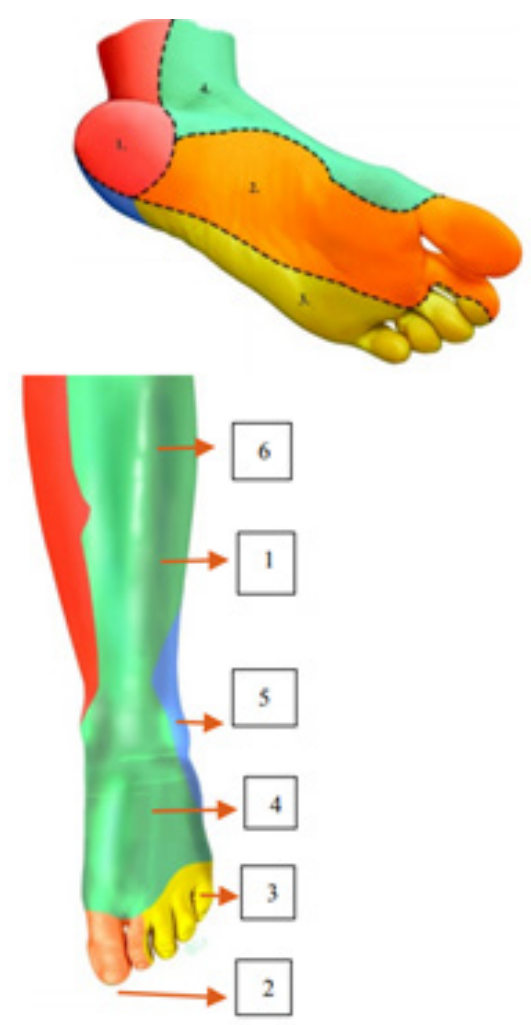

FIGURA 3 Angiossomos dos pés e tornozelos (modificado de Attinger $^{28}$, Alexandrescu ${ }^{25}$

- Ilustração de Iriam Starling

1. artéria tibial posterior

2. artéria medial plantar

2. artéria medial plantar

4. artéria dorsal do pé

5. artéria peroneal

6. artéria tibial anterior

\section{METODOLOGIA}

A partir das referências anatômicas estudadas por Taylor e Palmer ${ }^{5}$ em 1987, propõe-se a análise dos termogramas plantares dividindo-os em seis áreas de interesse de acordo com os angiossomos. Assim pode-se estudar o território da artéria medial plantar em três ROls correspondentes ao hálux, $1^{\circ}$ metatarso, arco plantar: o território da artéria plantar lateral através dos ROls do $5^{\circ}$ Metatarso e borda lateral do pé e o território da artéria tibial posterior na região do calcâneo (Figura 4). Termogramas adicionais podem ser colhidos no dorso dos pés para avaliação da artéria dorsal do pé e complementação do exame do território da artéria medial plantar com o exame do hálux e do território da artéria lateral plantar com o exame dos demais pododáctilos. Como o sistema simpático é responsável pelo controle vasomotor dos pés, pode-se realizar o teste provocativo com frio com a finalidade de realçar as alterações encontradas tanto qualitativamente, quanto quantitativamente, através da análise comparativa entre os dois pés ou utilizando o valor de temperatura esperado para a região, se não houver o membro contralateral normal que favoreça a comparação. Além disto, pode-se avaliar as diferenças de temperatura entre os artelhos para identificar anisotermias presentes em patologias neurológicas, vasculares ou reumatológicas. 7,9,19,20

Durante o período de março de 2015 e fevereiro de 2017, foram avaliados 22 pacientes recrutados por procura espontânea ao consultório, portadores de onicomicose crônica. O registro das imagens infravermelhas foi efetuado com câmera térmica Flir ${ }^{\circledR}$ modelo T420bx, FOL 18mm, resolução IR320x240, sob condições de controle de circulação de $\operatorname{ar}(<0,2 \mathrm{~ms})$, temperatura ambiente de $23^{\circ} \mathrm{C}$. Os termogramas foram colhidos das plantas dos pés com o paciente sentado ou ajoelhado. Foi feita a determinação das áreas anatômicas da planta dos pés com o objetivo de avaliar o comprometimento ortopédico/ neurológico/vascular das regiões e usar como auxilio diagnóstico. 


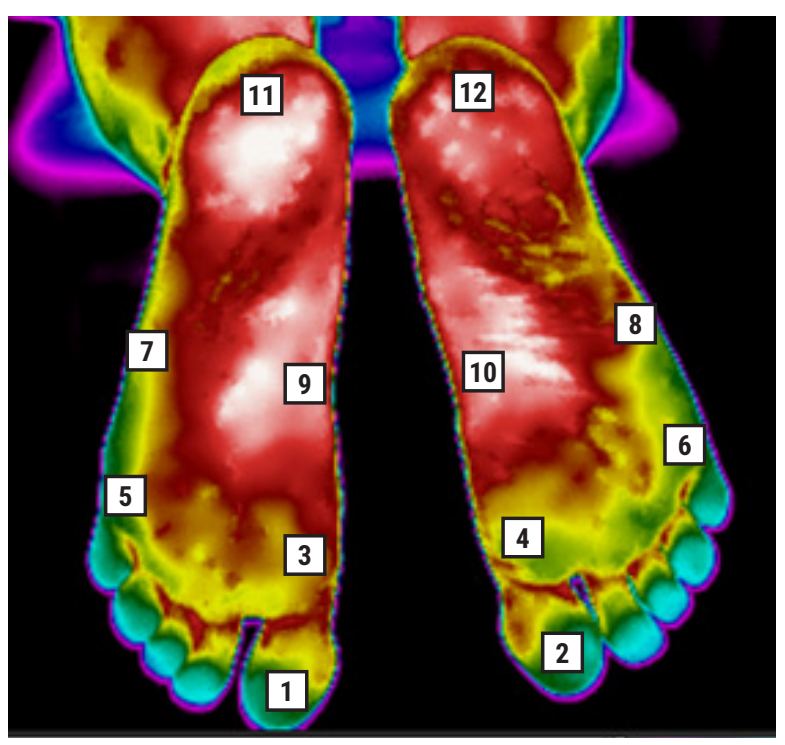

FIGURA 4 áreas de interesse (ROI) na região plantar

1. hálux esquerdo

2. hálux direito

3. $\mathbf{1}^{\circ}$ metatarso esquerdo

4. $\mathbf{1}^{\circ}$ metatarso direito

5. $5^{\circ}$ metatarso esquerdo

6. $5^{\circ}$ metatarso direito

7. lateral plantar esquerda

8. lateral plantar direita

9. medial plantar esquerda

10. medial plantar direita

11. calcâneo esquerdo

12. calcâneo direito

\section{RESULTADOS}

Os termogramas dos 22 pacientes foram analisados com o estudo das 6 ROIs plantares e comparados os valores de temperatura máxima, média e mínima das plantas do pé esquerdo (números ímpares) com o direito (números pares), com suas correspondentes variações de temperatura $(\triangle T)$.

Uma análise epidemiológica simples sobre a distribuição de OSDL(onicomicose superficial distal e lateral) e ODT (onicomicose distrófica total) por sexo e presença ou não de diabetes indicou que o diabetes se apresenta como um fator que aumenta o risco de onicomicose subungueal distal lateral (OR=8,33, com intervalo de confiança $=0,776-89,469$, não significativo) e atua como fator de proteção para onicomicose distrófica total $(\mathrm{OR}=0,156$, com intervalo de confiança=0,015-1,653, não significativo). Além disto, não houve diferenças dos $\Delta T$ (diferencial térmico entre 2 ROIs) de temperatura por sexo.

Quando se analisa o $\Delta T$ (variação de temperatura) da região do calcâneo (ROIs 11 e 12, Tabela 1) observa-se diferenças entre os grupos com e sem diabetes. A análise dos $\Delta T$ (variação de temperatura) da região do hálux (ROls 1 e 2, Tabela 2) apresentou diferenças significativas entre os grupos com e sem atividade física (praticantes regulares de corrida ou dança). Observou-se também $\Delta T$ significativo quando se analisa o ROI do hálux com e sem onicomicose superficial distal e lateral (Tabela 3). Não há diferenças nos Deltas de temperatura nos ROls selecionados quando se analisa os grupos com e sem ODT.

TABELA $1 \Delta T$ (variação de temperatura) dos ROls correspondentes à região do calcâneo.

\begin{tabular}{|r|r|r|r|}
\hline & $\Delta \mathrm{T} 11-12 \mathrm{max}$ & $\Delta \mathrm{T} 11-12 \mathrm{me}$ & $\Delta \mathrm{T} 11-12 \mathrm{~min}$ \\
\hline Mann-Whitney U & 20,500 & 25,500 & 34,500 \\
\hline Wilcoxon W & 156,500 & 161,500 & 170,500 \\
\hline $\mathrm{Z}$ & $-2,030$ & $-1,664$ & $-0,997$ \\
\hline P valor & 0,042 & 0,096 & 0,319 \\
\hline Sig exata [2*(Sig. de 1-cauda)] & $0,040 \mathrm{~b}$ & $0,098 \mathrm{~b}$ & $0,329 \mathrm{~b}$ \\
\hline
\end{tabular}

Calcâneo direito - área 12

Calcâneo esquerdo - área 11

max - máxima

me - média

min - mínima

TABELA $2 \Delta T$ dos ROls dos grupos com e sem atividade física correspondentes ao hálux direito e esquerdo - diferença de temperatura das áreas 1 e 2( $\Delta T$ 1-2), máxima (max), média (me) e min (mínima)

\begin{tabular}{|r|r|r|r|}
\hline & $\Delta \mathrm{T} 1-2 \mathrm{max}$ & $\Delta \mathrm{T} 1-2 \mathrm{me}$ & $\Delta \mathrm{T} 1-2 \mathrm{~min}$ \\
\hline Mann-Whitney U & 46,500 & 31,500 & 20,500 \\
\hline Wilcoxon W & 74,500 & 59,500 & 48,500 \\
\hline $\mathrm{Z}$ & $-0,427$ & $-1,484$ & $-2,260$ \\
\hline P valor & 0,670 & 0,138 & 0,024 \\
\hline Sig exata [2*(Sig. de 1-cauda)] & $0,680 \mathrm{~b}$ & $0,142 \mathrm{~b}$ & $0,021 \mathrm{~b}$ \\
\hline
\end{tabular}

Hálux direito - área 2

Hálux esquerdo - área 1

max - máxima

me - média

min - mínima 
TABELA 3 Diferenças nos $\Delta T$ no hálux direito e esquerdo ( $\Delta T$ 1-2) quando se analisa os grupos com e sem OSDL.

\begin{tabular}{|r|r|r|r|}
\hline & $\Delta \mathrm{T} 1-2 \mathrm{max}$ & $\Delta \mathrm{T} 1-2 \mathrm{me}$ & $\Delta \mathrm{T} 1-2 \mathrm{~min}$ \\
\hline Mann-Whitney $\mathrm{U}$ & 29,000 & 46,000 & 42,500 \\
\hline Wilcoxon $\mathrm{W}$ & 95,000 & 112,000 & 108,500 \\
\hline $\mathrm{Z}$ & $-2,086$ & $-0,0955$ & $-1,184$ \\
\hline P valor & 0,037 & 0,340 & 0,236 \\
\hline Sig exata [2*(Sig. de 1-cauda)] & $0,040 \mathrm{~b}$ & $0,365 \mathrm{~b}$ & $0,243 \mathrm{~b}$ \\
\hline
\end{tabular}

\section{Hálux direito - área 2}

Hálux esquerdo - área 1

max - máxima

me - média

min - mínima

\section{DISCUSSÃO}

Embora Sirgurgeirsson (2010), Scher (2007) e Ghannoum (2000) em estudos amplos 21,22,23 indiquem uma predominância de pacientes idosos, neste trabalho no qual examinou-se exclusivamente pacientes com onicomicose crônica, não se observou esta predominância pois a patologia esteve presente em adultos entre 34 e 86 anos, desta forma, a idade não foi fator determinante. ${ }^{30} \mathrm{O}$ estudo de Nagase (2001) indica que os pés ${ }^{24}$ considerados normais apresentam uma área de hiperradiação na região central da planta dos pés em formato de borboleta (butterfly patern); os pacientes avaliados aqui não apresentavam este padrão, mas irregularidade no exame qualitativo sugestivo de neuropatia periférica, com anisotermia entre as ROls e alterações vasculares (áreas hipo ou hiperradiantes) localizadas nos angiossomos estudados. Ressalta-se que a neuropatia distal simétrica pode acometer fibras finas vasomotoras e ter várias causas, entre estas o diabetes, abuso de bebidas alcóolicas, deficiência de vitamina B12 ou folato, e, ainda patologias hematológicas. O cold stress test foi realizado em 3 pacientes com o objetivo aumentar a sensibilidade do método para detectar disfunção de fibras finas vasomotoras em pacientes diabéticos, contudo este teste pode ser estendido para todos os portadores de onicomicose crônica a serem avaliados no futuro com o objetivo de detectar e tratar precocemente e melhorar as condições vasomotoras dos membros inferiores, assim como fazer o diagnóstico diferencial com problemas de origem ortopédica (congênitos ou decorrente de sobrecarga) ou vascular.

O exame por imagem infravermelha pode avaliar as plantas dos pés e auxiliar no diagnóstico da neuropatia distal simétrica nos seus estágios iniciais, assim como a disfunção endotelial e problemas circulatórios ou ortopédicos. Pode ser utilizado no acompanhamento para o tratamento com laser Nd-YAG 1064nm (Clearsens ${ }^{\circledR}$ Sciton ${ }^{\circledR}$ ) pois evidencia a recuperação da microcirculação (seja por melhora do controle vasomotor ou redução do processo inflamatório local). ${ }^{30}$

Os trabalhos de Alexandrescu, Wagner, Netten, Bagavathiappan avaliam a vascularização plantar ${ }^{25,16,9,10} \mathrm{com}$ finalidade de tratamento de reperfusão e enfatizam o exame da região do antepé, correspondentes aos ROls 1,3,5 (hálux, $1^{\circ}$ Metatarso e $5^{\circ}$ metatarso) e territórios anatômicos distais das artérias medial e lateral plantar. O estudo de Macdonald analisou 33 hot spots nos pés ${ }^{26}$ fazendo um mapa detaIhado com a finalidade de identificar áreas de inflamação comparando com o pé contralateral. Quesada, por outro lado, reforça a necessidade de um mínimo de 25 pixels na área estudada (ROI) pois o tamanho do ROI influenciaria a mensuração da temperatura, recomendando então ROIs maiores para reduzir a possibilidade de distorção da temperatura nos hot spots que medem apenas pontos. ${ }^{27}$

Embora a amostra do presente estudo seja pequena, sugere a necessidade do estudo das demais ROls plantares, de acordo com os angiossomas estudados por Taylor e lida ${ }^{5,18}$, e os termogramas de 
Nagase ${ }^{24}$ estendendo o estudo a toda a extensão da área vascularizada pelas artérias plantares medial e lateral, além da artéria tibial posterior, em 6 ROls plantares, levando em conta as possíveis comunicações arteriovenosas através de um exame não invasivo de fácil execução, inclusive à beira do leito.

\section{CONCLUSÃO}

O exame por imagem infravermelha pode servir de ferramenta para a detecção precoce de alterações vasculares, ortopédicas e neurológicas numa fase que são passíveis de tratamento e favorecendo a prevenção de uma evolução desfavorável e/ou complicações tardias. Aos pacientes cirúrgicos vasculares, orienta a viabilidade de estruturas anatômicas e consequentemente o planejamento cirúrgico pois auxilia na avaliação da circulação periférica e do tônus vasomotor. Nos pacientes com onicomicose crônica mostrou-se útil para rastrear e avaliar as co-morbidades sistêmicas ou locoregionais.

\section{AGRADECIMENTOS}

Iriam Starling - elaboração das ilustrações deste artigo, modificadas de Attinger 2006, Alexandrescu 2008, Suami 2003.

Julia de Sá e Souza - revisão e correção do texto em português

\section{REFERÊNCIAS}

1. Medeiros CR, Brioschi ML, Souza SN, Teixeira MJ. Infrared thermography to diagnose and manage venomous animal bites and stings. Rev Soc Bras Med Trop 2017; 50(2):1-5

2. Ring EFJ, Ammer K. Infrared thermal imaging in medicine. Physiol. Meas. 2012;33: R33-46.

3. Ammer K. The sensitivity of infrared imaging for diagnosis Raynaud'a phenomenon and for Thoracic Outlet Syndrome is dependent on the method of temperature extraction from thermal images. Thermol. Int. 2008; 18 81-8

4. Ammer K. Cold challenge to provoke a vasospastic reaction in fingers determined by temperature measurements; a systematic review. Thermol. Int. 2009; 19:109-18.

5. Taylor, GI, Pan, WR. Angiossomes of the legs: Anatomic Study and Clinical Implications. Plastic Reconst Surg. 1998, 102(3):599-616.

6. Schalin L. Arteriovenous communications in varicose veins localized by thermography and identified by operative microscopy.1981, 147(6):409-420

7. Balbinot LF, Canani $L H$, Robinson CC, Achaval M, Zaro MA, Brioschi ML. Plantar Termography is useful in the early diagnosis of diabetic neuropathy. Clinics 2012; 67(12):1419-25

8. Balbinot LF, Robinson CC, Achaval M, Zaro MA, Brioschi $M L$. Repeatability of infrared plantar thermography in diabetes patients: A Pilot Study. J Diabetes Sci Technol 2013;7(5):1130-37

9. Netten JJ, Baal JG, Liu C, Heijden F, Bus SA. Infrared thermal imaging for automated detection of diabetic foot complications. J Diabetes Sci Technol 2013;7(5): 1122-29

10. Bagavathiappan S, Jayakumar T, Someshwar PN, Varalakshmi M, Mohan V. Correlation between plantar foot temperature and diabetic neuropathy: A case study by using and infrared thermal imaging technique. $J$ Diabetes Sci Technol 2010; 4(6):1386-92.

11. Kambiz S, Neck JW, Cosgun SG, Velzen MHN, Janssen JAMJL, Avazverdi N, Houvius SER, Walbeehm ET. An Early diagnostic tool for diabetic peripheral neuropathy in rats. PLoS One 2015; 10(5): e0126892.

12. Goldman, MP. Sclerotherapy: Treatment of varicose and telangiectatic leg veins - 5th ed. 2011, Elsevier pg 4

13. Braga,RZM, Evangelista AL, Santos Filho SJA, Trigo EL, Carnevali Junior LC. Mecanismos bioquímicos da contração muscular promovida pela cãibra. Rev Bras Fisiol Exerc 2014, 13 (4):231-38.

14. Weiss R, Feied CF, Weiss MA. Venous Physiology an Pathophysiology in Vein diagnosis and treatment: 
A compreensive Approach. 2001, Chapter 4: 411-549. McGraw-Hill

15. Lima RCM, Santiago L, Moura RMF, Filaretti FAS, Souza CSA, Evangelista SSM, Britto RR. Efeitos do fortalecimento muscular da panturrilha na hemodinâmica venosa e na qualidade de vida em um portador de insuficiência venosa crônicaJ Vasc Br 2002; 1(3):219-26.

16. Wagner Jr FW. The dysvascular foot: a system for diagnosis and treatment. Foot Ankle 1981; 2: 64-122.

17. Teixeira MJ, Lin TY, Fernandes TD, Hernandez AJ, Romano MA, Forni JEN, Amatuzzi M. Lower limb pain. Rev Med 2001;80(ed esp pt 2):391-414.

18. Iida O, Nanto S, Uematsu M, Ikeoka K, Okamoto S, Dohi T, Fujita M, Terashi H, Nagata S. Importance of the Angiosome Concept for Endovascular Therapy in Patients with Critical Limb Ischemia. 2010 Cathet Cardiov Interv 75:830-836

19. Staffa E, Bernard V, Kubicek L, Vlachovsky R, VLK D, Mornstein V, Bourek A, Staffa R. Infrared thermography as option for evaluating the treatment effect of percutaneous transluminal angioplasty by patients with peripheral arterial disease vascular. 2017 25(1):42-49.

20. Lerkvaleekul B, Jaovisidha S, Sungkarat W, Chitrapazt N, Fuangfa P, Ruangchaijatuporn T, Vilaiyuk $S$, The Comparisons between thermography and ultrasonography with physical examination for wrist joint assessment in juvenile idiopathic arthritis. Physiol Meas 2017 38(5):691-700.

21. Sigurgeirsson B. Prognostic factors for cure following treatment of onychomycosis. J Eur Acad Dermatol Venerol. 2010; 24:679-84.

22. Ghannoum MA, Hajjeh RA, Scher R, Konnikov N, Gupta AK, Summerbell R, Sullivan S, Daniel R, Krusinski P, Fleckman P, Rich P, Odom R, Aly R, Parise D, Zaiac M, Rebell G, Lesher J, Gerlach B. A large-scale north American study of fungal isolates from nails: the frequency of onychomycosis, fungal distribution, and antifungal susceptibility patterns. J Am Acad Dermatol. 2000;43(4): 641-8

23. Pariser D, Elewski B, Rich P, Scher RK. Update on onychomycosis: effective strategies for diagnosis and treatment. Semin Cutan Med Surg. 2013:32(2s):1-13.

24. Nagase T, Sanada H, Takehara K, Oe M, lizaka S, Ohashi Y, Oba M, Kadowaki T, Nakagami G. Variations of plantar thermographic patterns in normal controls and non-ulcer diabetic patients: Novel classification using angiosome concept. J Plast, Reconst Aest Surg 2001 64, 860-866.

25. Alexandrescu VA, Hubermont G, Philips Y, Guillaumie B, Ngongang C, Vandenbossche P, Azadad K, Ledent G, Horion J. Selective Primary Angioplasty Following an Angiosome Model of Reperfusion in the Treatment of Wagner 1-4 Diabetic Foot Lesions: Practice in a Muldisciplinary Diabetic Limb Service. J Endovasc Ther
2008; 15:580-593.

26. Macdonald A, Petrova N, Ainarkar S, Allen J, Plassman P, Whittam A, Bevans J, Ring F, Kluwe B, Simpson R, Rogers L, Machin G, Edmonds M. Thermal symmetry of healthy feet: a precursor to a thermal study of diabetic feet prior to skin breakdown. Physiol. Meas. 2017; 38:33-44.

27. Quesada JJP. Methodological aspects of infrared thermography in humam assessment in chapter3. Application of Infrared Thermography in Sports Science, Biological and Medical Physics, Biomedical Engineering. 2017. Springer International; 49-79.

28. Attinger CE, Evans KK, Bulan E, Blume P, Cooper P. Angiossomes of the Foot and Ankle and Clinical Implications for Limb Salvage: Reconstruction, Incisions, and Revascularization. Plast Reconstr Surg2006; 117(suppl):261S

29. Suami H, Taylor GI, Pan WR. Angiosome Territories of the Nerves of the Lower Limbs. Plast Reconstr Surg 2003; 112:1790-1798

30. Vila TVM, Rosental S, de Sá Guimarães CMD. A new model of in vitro fungal biofilms formed on human nail fragments allows reliable testing of laser and light therapies against onychomycosis. Laser Med Sci. 2014;30(3):1031-9 